\title{
Hinterm Horizont ... Herzliche Einladung zum Deutschen Kongress für Orthopädie und Unfallchirurgie DKOU 2015
}

\author{
Beyond the Horizon ... Invitation to the DKOU 2015
}

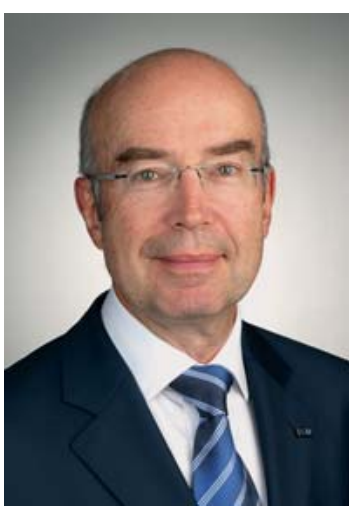

M. Nerlich
Der DKOU mit ca. 12000 Teilnehmern hat sich zum größten medizinischen Fachkongress in Europa und zu einem der größten Orthopädieund Traumatologie-Kongresse weltweit entwickelt. Mit unserem Motto „Hinterm Horizont“ möchten wir die Perspektiven erweitern - über den Tellerrand blicken.

Der DKOU muss als der zentrale Kongress für unser Fach allen Interessenslagen gerecht werden und seine Rolle als die führende Veranstaltung innerhalb einer ausufernden Kongresslandschaft wahrnehmen.

Der DKOU will Orientierung bieten: Die Jüngeren brauchen eine verlässliche Übersicht über alle Bereiche unseres breiten Faches, der Fortgeschrittene wünscht sich die Vermittlung einer differenzierteren Sichtweise. Der Fachmann erhält den aktuellsten Stand für seinen persönlichen Benchmark. An diesem bewährten Muster des Kongressaufbaues wird festgehalten. Die Sektionen und Arbeitsgruppen haben genug Möglichkeiten, sich am Dienstag intern auszutauschen, aber auch Interessierte durch attraktive Darstellungen ihrer Spezialitäten anzulocken. Während der Mittwoch schwerpunktmäßig den Unfallchirurgen und der Donnerstag den operativ tätigen Orthopäden interessieren dürfte, ist der Freitag vornehmlich dem konservativ tätigen Fachmann gewidmet. Wir haben in mühevoller Kleinarbeit versucht, für jede Interessenslage in sich schlüssige „Tracks“ zusammenzustellen, in denen sich die Spezialitäten des Faches wiederfinden können.

Die „Blockbuster“ dürften wieder die „Expertenrunden“ und „Tipps und Tricks“-Sitzungen werden, die unsere alltäglichen Herausforderungen adressieren.

Besonders hervorzuheben ist die Aufmerksamkeit, die dem Nachwuchs in 0 \& U gilt. Spezielle
Vortragssitzungen gehen auf die Fragen und Bedürfnisse der Ärztinnen und Ärzte in Fachweiterbildung ein. So wurden die Seminare in zwei Wissensebenen aufgeteilt, eine Reihe - erstmals gemeinsam O \&U von Kopf bis Fuß - für den jüngeren Assistenten (Basics) und eine Reihe für den fortgeschrittenen, facharztnahen Weiterbildungsassistenten. Darüber hinaus ist der DKOU eine wunderbare Bühne für unsere ambitionierten „High Potentials“, um sich wissenschaftliche Sporen zu verdienen. Geeignete Auszeichnungen werden einen entsprechenden Ansporn bieten.

Die Themenvielfalt des Kongresses spiegelt die Breite unseres Faches wider und lässt keine Wünsche offen. So kann jeder sich in seiner Spezialität vertiefen, oder auch mal über den Tellerrand schauen und erfahren, was unser Fach sonst noch so alles zu bieten hat. Gerade die berufspolitischen Themen betreffen „heiße Eisen“, bei denen es um die Zukunft unseres Faches geht.

Das hohe wissenschaftliche Niveau des DKOU hat sich mittlerweile in der Welt herumgesprochen. Viele ausländische Kollegen sind aufmerksam geworden und haben Interesse an einer Teilnahme gezeigt. Ziel der Erweiterung des internationalen Programms ist die Intensivierung des wissenschaftlichen Dialogs mit unseren nicht deutschsprechenden Kollegen in Europa und darüber hinaus. Dafür gibt es durchgehend englischsprachige Sitzungen, in die führende internationale Fachgesellschaften wie die ORS, EORS, EHS, OTA und andere aktiv in unser wissenschaftliches Programm eingebunden sind. Die Anerkennung unserer wissenschaftlichen und klinischen Standards ist uns Ansporn für einen fachlich und persönlich großartigen Kongress 2015.

Ihr Michael Nerlich

\author{
Bibliografie \\ DOI http://dx.doi.org/ \\ 10.1055/s-0035-1558130 \\ Z Orthop Unfall 2015; 153: 478 \\ (c) Georg Thieme Verlag KG \\ Stuttgart · New York · \\ ISSN 1864-6697 \\ Korrespondenzadressen \\ Prof. Dr. Michael Nerlich \\ Direktor der Klinik für \\ Unfallchirurgie \\ Universitätsklinikum Regensburg \\ Franz-Josef-Strauß-Allee 11 \\ 93042 Regensburg \\ Tel.: 0941/9446805 \\ Fax: 0941/9446806 \\ michael.nerlich@ukr.de
}

